Supplement of Biogeosciences, 14, 5741-5752, 2017

https://doi.org/10.5194/bg-14-5741-2017-supplement

(C) Author(s) 2017. This work is distributed under

the Creative Commons Attribution 3.0 License.

(c) (1)

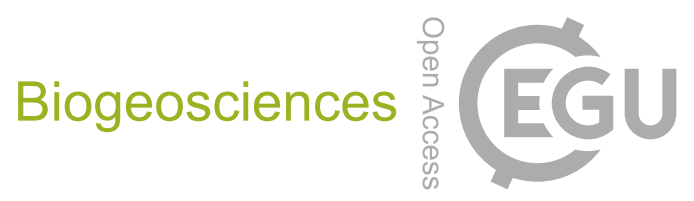

Supplement of

\title{
Impact of diurnal temperature fluctuations on larval settlement and growth of the reef coral Pocillopora damicornis
}

\section{Lei Jiang et al.}

Correspondence to: Pei-Yuan Qian (boqianpy@ust.hk) and Hui Huang (huanghui@ scsio.ac.cn)

The copyright of individual parts of the supplement might differ from the CC BY 3.0 License. 


\section{Supplementary figures}
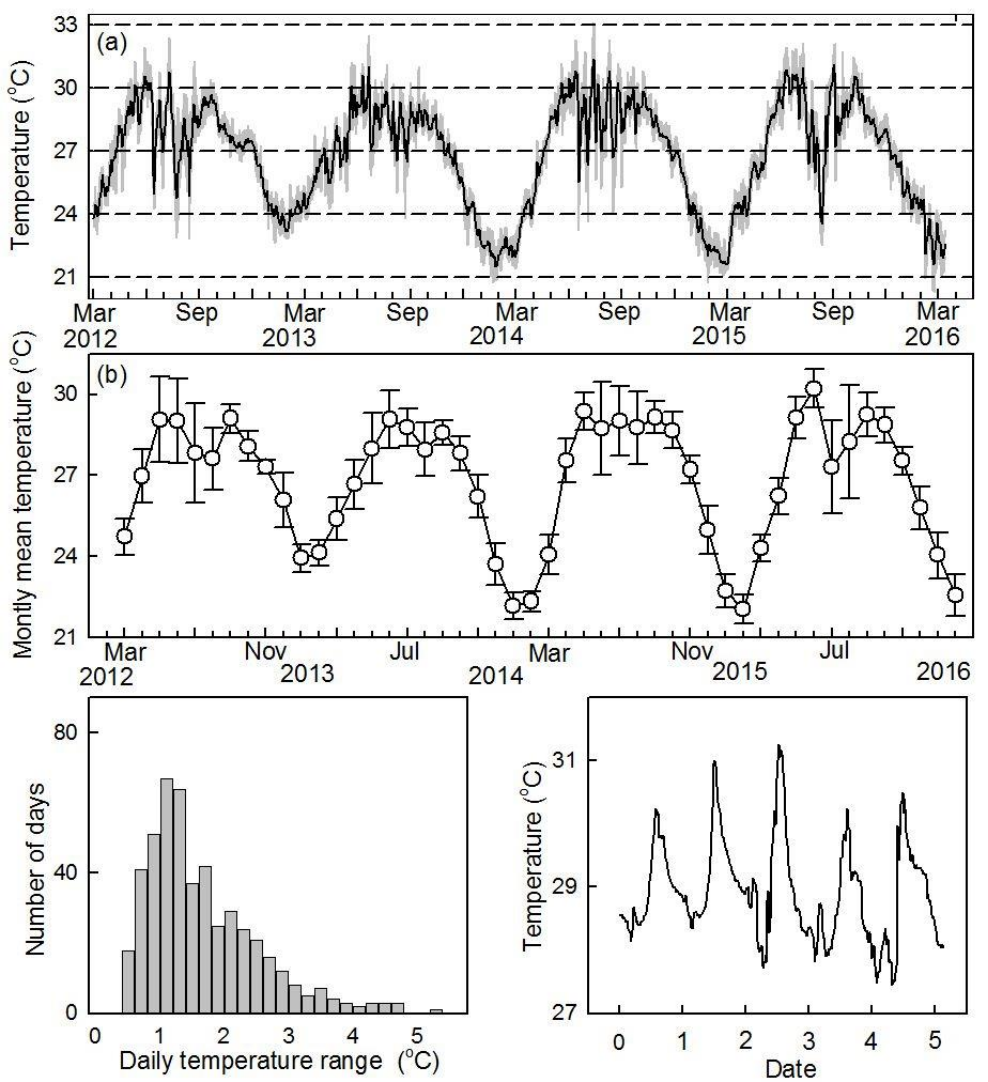

Fig S1. Temperature data at $3 \mathrm{~m}$ depth on Luihuitou fringing reef from 2012 to 2016.

(a) The bold black line shows the daily average temperatures, and the shaded grey area illustrates the daily maximum and minimum temperatures; (b) monthly mean ( \pm SD) temperatures from 2012 to 2016 ; (c) histogram of daily temperature range in summer (June-September) from 2012 to 2015; (d) showing a period of 5 days of diurnal temperature fluctuations $\left(\sim 3^{\circ} \mathrm{C}\right)$ in June 2014 . 
Fig S2. Metamorphosed and free drifting larvae of Pocillopora damicronis with extended tentacles.

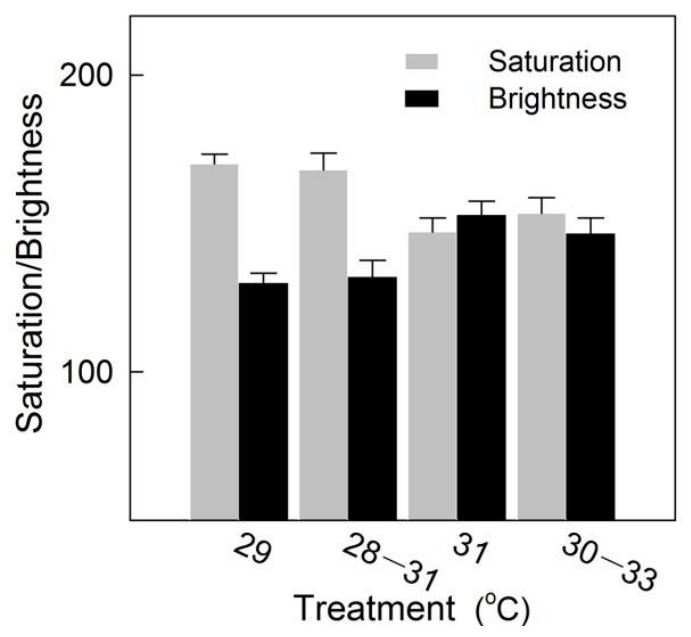

Fig S3. Photographic metrics for Pocillopora damicornis recruits at different temperature treatments. 

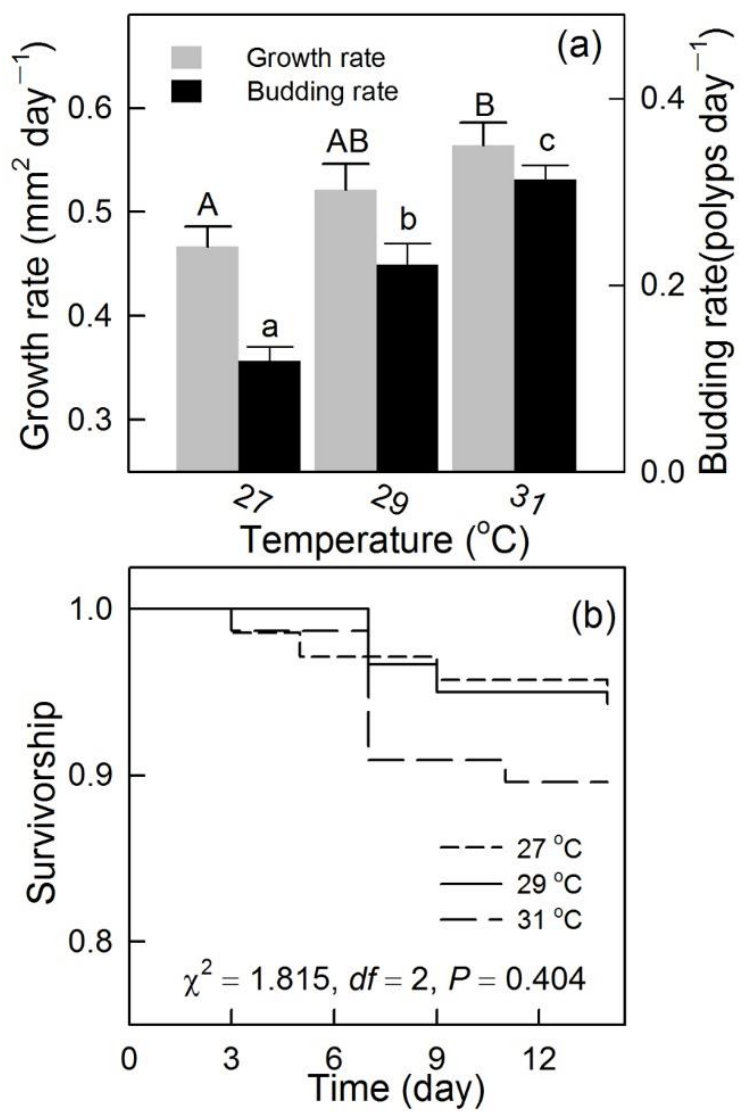

Fig S4. (a) Growth and (b) survival of Pocillopora damicornis recruits after 2 weeks at 27,29 and $31^{\circ} \mathrm{C}$. Lateral growth and budding rates were measured as described in Materials and methods. Growth was analyzed with one-way ANOVA followed by Fisher's LSD multiple comparisons (as indicated by letters). Survival curves were compared among treatments with Kaplan-Meier (KM) log-rank analysis. 


\section{Supplementary tables}

Table S1. Statistical results of two-way ANOVAs on the effects of temperature treatments on larval survival and settlement of Pocillopora damicornis.

\begin{tabular}{llcccc}
\hline Variables & Source of variation & $d f$ & MS & $F$ & $P$ \\
\hline Settlement assays & & & & & \\
Larval survival & Temp level & 1 & 0.003 & 3 & 0.11 \\
& Temp regime & 1 & 0.003 & 3 & 0.11 \\
& Level*Regime & 1 & 0.003 & 3 & 0.11 \\
& Error & 12 & 0.001 & & \\
Floating \& Metamorphosed & Temp level & 1 & 0.041 & 1.64 & 0.22 \\
& Temp regime & 1 & 0.008 & 0.32 & 0.58 \\
& Level*Regime & 1 & 0.040 & 1.59 & 0.23 \\
& Error & 12 & 0.025 & & \\
Swimming larvae & Temp level & 1 & 0.038 & 0.568 & 0.47 \\
& Temp regime & 1 & 0.006 & 0.094 & 0.76 \\
& Level*Regime & 1 & 0.038 & 0.568 & 0.47 \\
Settlement success & Error & 12 & 0.067 & & \\
& Temp level & 1 & 0.133 & 9.43 & $\mathbf{0 . 0 1}$ \\
& Temp regime & 1 & 0.043 & 3.06 & 0.11 \\
& Level*Regime & 1 & 0.057 & 4.00 & 0.06 \\
& Error & 12 & 0.014 & & \\
\hline
\end{tabular}

Table S2. Pairwise post-hoc multiple comparisons (Fisher's LSD) for larval settlement. $P$ values in bold indicate significance level at $\alpha=0.05$.

\begin{tabular}{|l|l|l|l|l|}
\cline { 2 - 5 } \multicolumn{1}{l|}{ Settlement } & $29{ }^{\circ} \mathrm{C}$ & $28-31{ }^{\circ} \mathrm{C}$ & $31^{\circ} \mathrm{C}$ & $30-33^{\circ} \mathrm{C}$ \\
\hline $29{ }^{\circ} \mathrm{C}$ & & & & \\
\hline $28-31^{\circ} \mathrm{C}$ & 0.861 & & & \\
\hline $31^{\circ} \mathrm{C}$ & $\mathbf{0 . 0 0 4}$ & $\mathbf{0 . 0 0 6}$ & & \\
\hline $30-33{ }^{\circ} \mathrm{C}$ & 0.374 & 0.471 & $\mathbf{0 . 0 2 2}$ & \\
\hline
\end{tabular}


Table S3. Statistical results of two-way repeated measures ANOVAs on the effects of temperature treatments on $\mathrm{Fv} / \mathrm{Fm}$ and $\Delta \mathrm{F} / \mathrm{F}_{\mathrm{m}}$ ' of juvenile Pocillopora damicornis.

\begin{tabular}{llllll}
\hline Source of variation & $\mathrm{SS}$ & $d f$ & $\mathrm{MS}$ & $F$ & $P$ \\
\hline $\boldsymbol{F v}$ /Fm & & & & & \\
Within subjects & & & & & \\
Day & 0.129 & 3 & 0.043 & 98.83 & $<\mathbf{0 . 0 0 1}$ \\
Day*Temp level & 0.003 & 3 & 0.001 & 2.468 & 0.063 \\
Day*Temp regime & 0.005 & 3 & 0.002 & 4.142 & $\mathbf{0 . 0 0 7}$ \\
Day*level*regime & 0.005 & 3 & 0.002 & 3.928 & $\mathbf{0 . 0 0 9}$ \\
Between subjects & & & & & \\
Temp level & 0.058 & 1 & 0.058 & 69.27 & $<\mathbf{0 . 0 0 1}$ \\
Temp regime & 0.004 & 1 & 0.004 & 4.384 & $\mathbf{0 . 0 4 0}$ \\
Level*Regime & 0.003 & 1 & 0.003 & 3.771 & 0.056 \\
AF/F ${ }^{\prime}$ & & & & & \\
Within subjects & & & & & \\
Time & 0.193 & 3 & 0.064 & 58.88 & $<\mathbf{0 . 0 0 1}$ \\
Time *Temp level & 0.024 & 3 & 0.008 & 7.228 & $<\mathbf{0 . 0 0 1}$ \\
Time *Temp regime & 0.014 & 3 & 0.005 & 4.319 & $\mathbf{0 . 0 0 6}$ \\
Time *level*regime & 0.051 & 3 & 0.017 & 15.44 & $<\mathbf{0 . 0 0 1}$ \\
Between subjects & & & & & \\
Temp level & 0.015 & 1 & 0.015 & 3.146 & 0.082 \\
Temp regime & 0.055 & 1 & 0.055 & 11.44 & $\mathbf{0 . 0 0 1}$ \\
Level*Regime & $<0.001$ & 1 & $<0.001$ & $<0.001$ & 0.994 \\
\hline & & & & &
\end{tabular}


Table S4. Statistical results of separated two-way ANOVAs on the effects of temperature treatments on maximum quantum yield (Fv/Fm) and effective quantum yield $\left(\Delta \mathrm{F} / \mathrm{F}_{\mathrm{m}}{ }^{\prime}\right)$ of juvenile Pocillopora damicornis at a specific measuring time point.

\begin{tabular}{|c|c|c|c|c|c|c|}
\hline Day/time & Source of variation & SS & $d f$ & MS & $F$ & $P$ \\
\hline \multicolumn{7}{|l|}{$F v / F m$} \\
\hline \multirow[t]{4}{*}{ August 26} & Temp level & 0.010 & 1 & 0.010 & 18.34 & $<0.001$ \\
\hline & Temp regime & 0.007 & 1 & 0.007 & 12.63 & 0.001 \\
\hline & Level*Regime & $<0.001$ & 1 & $<0.001$ & 0.136 & 0.714 \\
\hline & Error & 0.043 & 76 & 0.001 & & \\
\hline \multirow[t]{4}{*}{ August 27} & Temp level & 0.007 & 1 & 0.007 & 16.44 & $<0.001$ \\
\hline & Temp regime & 0.001 & 1 & 0.001 & 2.121 & 0.149 \\
\hline & Level*Regime & 0.001 & 1 & 0.001 & 1.205 & 0.276 \\
\hline & Error & 0.033 & 76 & $<0.001$ & & \\
\hline \multirow[t]{4}{*}{ August 28} & Temp level & 0.023 & 1 & 0.023 & 48.23 & $<0.001$ \\
\hline & Temp regime & $<0.001$ & 1 & $<0.001$ & 0.739 & 0.393 \\
\hline & Level*Regime & $<0.001$ & 1 & $<0.001$ & 0.280 & 0.598 \\
\hline & Error & 0.037 & 76 & $<0.001$ & & \\
\hline \multirow[t]{4}{*}{ August 29} & Temp level & 0.021 & 1 & 0.021 & 31.33 & $<0.001$ \\
\hline & Temp regime & 0.001 & 1 & 0.001 & 0.977 & 0.326 \\
\hline & Level*Regime & 0.008 & 1 & 0.008 & 11.45 & 0.001 \\
\hline & Error & 0.050 & 76 & 0.001 & & \\
\hline \multicolumn{7}{|l|}{$\Delta F / F_{m}^{\prime}$} \\
\hline \multirow[t]{4}{*}{ 08:00 } & Temp level & 0.004 & 1 & 0.004 & 1.661 & 0.203 \\
\hline & Temp regime & 0.002 & 1 & 0.004 & 0.750 & 0.390 \\
\hline & Level*Regime & 0.038 & 1 & 0.038 & 14.29 & $<0.001$ \\
\hline & Error & 0.149 & 56 & 0.003 & & \\
\hline \multirow[t]{4}{*}{$11: 00$} & Temp level & 0.017 & 1 & 0.017 & 9.270 & 0.006 \\
\hline & Temp regime & 0.008 & 1 & 0.008 & 4.384 & 0.040 \\
\hline & Level*Regime & 0.003 & 1 & 0.003 & 3.771 & 0.056 \\
\hline & Error & & & & & \\
\hline \multirow[t]{4}{*}{$14: 00$} & Temp level & 0.011 & 1 & 0.011 & 6.301 & 0.015 \\
\hline & Temp regime & 0.023 & 1 & 0.023 & 13.06 & 0.001 \\
\hline & Level*Regime & 0.004 & 1 & 0.004 & 2.538 & 0.117 \\
\hline & Error & 0.098 & 56 & 0.002 & & \\
\hline \multirow[t]{4}{*}{$17: 00$} & Temp level & 0.018 & 1 & 0.018 & 11.20 & 0.001 \\
\hline & Temp regime & 0.038 & 1 & 0.038 & 23.71 & $<0.001$ \\
\hline & Level*Regime & 0.004 & 1 & 0.004 & 2.730 & 0.104 \\
\hline & Error & 0.090 & 56 & 0.002 & & \\
\hline
\end{tabular}


Table S5. Statistical results of two-way ANOVAs on the effects of temperature treatments on maximum excitation pressure $(\mathrm{Qm})$ and bleaching index and growth of juvenile Pocillopora damicornis.

\begin{tabular}{llcccc}
\hline Variables & Source of variation & $d f$ & MS & $F$ & $P$ \\
\hline Qm & Temp level & 1 & 0.001 & 0.137 & 0.713 \\
& Temp regime & 1 & 0.067 & 10.45 & $\mathbf{0 . 0 0 2}$ \\
& Level*Regime & 1 & $<0.001$ & 0.075 & 0.786 \\
Error & 56 & 0.006 & & \\
Bleaching & Temp level & 1 & 0.036 & 1.627 & 0.205 \\
& Temp regime & 1 & 0.007 & 0.328 & 0.568 \\
& Level*Regime & 1 & 0.014 & 0.636 & 0.427 \\
Budding rate & Error & 107 & 0.022 & & \\
& Temp level & 1 & 2.258 & 22.80 & $<\mathbf{0 . 0 0 1}$ \\
& Temp regime & 1 & 0.132 & 1.251 & 0.262 \\
Lateral growth & Level*Regime & 1 & 0.002 & 0.018 & 0.894 \\
& Error & 107 & 0.090 & & \\
& Temp level & 1 & 0.358 & 25.42 & $<\mathbf{0 . 0 0 1}$ \\
& Temp regime & 1 & 0.030 & 2.123 & 0.148 \\
& Level*Regime & 1 & 0.001 & 0.038 & 0.845 \\
& Error & 107 & 0.014 & & \\
& Temp level & 1 & 8221 & 36.70 & $<\mathbf{0 . 0 0 1}$ \\
& Temp regime & 1 & 1036 & 4.625 & $\mathbf{0 . 0 3 4}$ \\
& Level*Regime & 1 & 463.3 & 2.068 & 0.154 \\
& Error & 96 & 223.9 & & \\
\hline
\end{tabular}

Table S6. Pairwise post-hoc multiple comparisons (Fisher's LSD) for calcification. $P$ values in bold indicate significance level at $\alpha=0.05$.

\begin{tabular}{|l|l|l|l|l|}
\cline { 2 - 5 } \multicolumn{1}{l|}{ Calcification } & $29{ }^{\circ} \mathrm{C}$ & $28-31^{\circ} \mathrm{C}$ & $31{ }^{\circ} \mathrm{C}$ & $30-33^{\circ} \mathrm{C}$ \\
\hline $29^{\circ} \mathrm{C}$ & & & & \\
\hline $28-31^{\circ} \mathrm{C}$ & 0.625 & & & \\
\hline $31^{\circ} \mathrm{C}$ & $<\mathbf{0 . 0 0 1}$ & $<\mathbf{0 . 0 0 1}$ & & \\
\hline $30-33^{\circ} \mathrm{C}$ & $\mathbf{0 . 0 0 8}$ & $\mathbf{0 . 0 0 2}$ & $\mathbf{0 . 0 1 0}$ & \\
\hline
\end{tabular}

